\title{
Analysis of 7-tesla diffusion-weighted imaging in the prediction of pituitary macroadenoma consistency
}

\author{
John W. Rutland, BA, ${ }^{1,2}$ Joshua Loewenstern, BA, ${ }^{2}$ Daniel Ranti, BS, ${ }^{2}$ \\ Nadejda M. Tsankova, MD, PhD, ${ }^{4}$ Christopher P. Bellaire, BA, ${ }^{2}$ Joshua B. Bederson, MD, ${ }^{2}$ \\ Bradley N. Delman, MD, MS, ${ }^{1,3}$ Raj K. Shrivastava, MD, ${ }^{2}$ and Priti Balchandani, PhD ${ }^{1}$
}

${ }^{1}$ Translational and Molecular Imaging Institute; and Departments of ${ }^{2}$ Neurosurgery, ${ }^{3}$ Diagnostic, Molecular and Interventional Radiology, and ${ }^{4}$ Pathology, Icahn School of Medicine at Mount Sinai, New York, New York

\begin{abstract}
OBJECTIVE Endoscopic surgery is an effective treatment strategy for pituitary adenomas; however, intrinsic tumor properties such as tumor consistency can challenge or preclude gross-total resection. Preoperative characterization of tumor consistency may help to guide the surgical approach and to predict the extent of resection that is possible. Advanced radiological modalities such as 7T diffusion-weighted imaging (DWI) may be useful in probing biological tissue properties of pituitary adenomas. The objective of the present study was to examine 7T DWI as a novel method of measuring the consistency of pituitary adenomas.
\end{abstract}

METHODS Thirteen patients with pituitary macroadenomas underwent 7T MRI, including a DWI image acquisition. Tumor apparent diffusion coefficient (ADC) was normalized to the adjacent temporal gray matter ADC. All patients underwent resection, and a single neurosurgeon blinded to ADC values rated tumor firmness from 1 (least firm) to 5 (most firm) using objective criteria. The tumor specimens were evaluated histopathologically for cellularity, collagen content, and vascularity by a neuropathologist who was also blinded to ADC values. The tumor ADC was correlated with intraoperative consistency rating, histopathology, and extent of resection. Receiver operating characteristic (ROC) curve analyses were performed to identify thresholds to predict tumor consistency.

RESULTS Corrected ADC values were significantly correlated with both tumor firmness $(r=-0.60, p=0.029)$ and the extent of trichrome staining $(r=-0.72, p=0.009)$ such that greater ADC values were associated with both decreased tumor firmness and decreased collagen staining. Correlations between ADC values and tumor vascularity were not significant $(r=-0.09, p=0.78)$. Corrected ADC values in totally resected tumors (1.54) were greater than those in subtotally resected tumors $(0.85)(p=0.02)$, and ADC values were greater with moderate tumor cellularity $(1.51)$ than with high tumor cellularity $(0.8)(p=0.035)$. There was a trend-level association for partial resections to exhibit greater tumor firmness rating ( 3 vs $1.7 ; p=0.051)$. Finally, the degree of trichrome staining positively correlated with tumor firmness $(r=$ $0.60, p=0.04$ ). The optimal threshold for predicting intraoperative consistency rating was an ADC ratio of 0.87 (sensitivity $80 \%$, specificity $100 \%$, area under the curve [AUC] $0.90 ; p=0.043$ ). The optimal cutoff for distinguishing the extent of resection was 1.19 (sensitivity $85.7 \%$, specificity $83.3 \%$ AUC $0.833 ; p=0.046$ ).

CONCLUSIONS The authors' results suggest that a high-resolution ADC of pituitary adenomas is a sensitive measure of tumor consistency. 7T DWI may hold clinical value in the preoperative workup and surgical management of patients with pituitary macroadenomas.

https://thejns.org/doi/abs/10.3171/2019.12.JNS192940

KEYWORDS pituitary adenoma; 7-tesla MRI; 7T MRI; tumor consistency; endoscopic endonasal surgery; neurosurgery; diffusion-weighted imaging; pituitary surgery

$\mathrm{A}$ DVANCEMENTS in the field of endoscopic transsphenoidal surgery (ETS) have permitted high rates of gross-total resection of pituitary adenomas and have reduced the need for craniotomy-based approaches to the anterior skull base..$^{20}$ The utility of ETS has expanded to almost all types of pituitary adenomas; however, intrinsic tumor features such as firmer tumor consistency can hamper gross-total resection during ETS. 9,23,27,29,32 Hence, while the majority of pituitary adenomas are soft and can be adequately resected with curettage and aspiration, $5 \%-$

ABBREVIATIONS ADC = apparent diffusion coefficient; $A U C=$ area under the curve; $D W I=$ diffusion-weighted imaging; $E T S=$ endoscopic transsphenoidal surgery; FA = flip angle; FOV = field of view; ROC = receiver operating characteristic; ROI = region of interest; SNR = signal-to-noise ratio

SUBMITTED October 29, 2019. ACCEPTED December 30, 2019.

INCLUDE WHEN CITING Published online February 28, 2020; DOI: 10.3171/2019.12.JNS192940. 
$13 \%$ are considered fibrous and are challenging to remove endoscopically. ${ }^{7,27}$ Noninvasive radiological markers that can predict pituitary adenoma consistency are useful for surgical planning, predicting the extent of resection, and identifying cases in which transcranial surgery may be appropriate.

Prior studies have attempted to characterize the consistency of pituitary adenoma; however, the ability of preoperative MRI to reliably predict pituitary adenoma firmness is controversial. ${ }^{30}$ Thus, additional investigation is necessary to determine the predictive value of MRI for tumor consistency. Most previous studies have employed T2-weighted imaging in this effort, and while some studies have found that an attenuated T2-weighted signal intensity is associated with firmer tumors, ${ }^{14,27}$ others have failed to identify a significant correlation between MRI metrics and pituitary adenoma consistency., ${ }^{713}$

Recently, the ability of diffusion-weighted imaging (DWI) to probe pituitary tumor consistency has been studied, ${ }^{19,29,33}$ but incongruent findings across a small number of studies have limited the application of this sequence in clinical practice. Using 1.5T imaging, Pierallini et al. reported that softer pituitary adenomas had lower apparent diffusion coefficient (ADC) values than firmer tumors, such that tumors with restricted water diffusion were more easily resected..$^{23}$ Conversely, Boxerman et al. found that softer tumors had significantly greater ADC values than firmer tumors, also using $1.5 \mathrm{~T}$. ${ }^{9}$ Suzuki et al. also found that softer tumors had higher ADC values than tumors of an intermediate consistency, but this effect did not reach statistical significance. ${ }^{29}$

The purpose of the present study was to apply 7T DWI to determine whether a tumor ADC can be used as a reliable preoperative predictor of pituitary adenoma consistency. Since prior studies have shown discordant results at lower field strengths, the higher signal-to-noise ratio (SNR) and spatial resolution attainable with 7T MRI may provide useful data that can clarify the relationship between tissue diffusion properties and pituitary macroadenoma consistency.

\section{Methods \\ Participants}

The Institutional Review Board of the Icahn School of Medicine at Mount Sinai approved this study before participant recruitment. Thirteen patients with pituitary adenomas were recruited through their neurosurgeon (R.K.S.) at the Mount Sinai Medical Center between September 2014 and August 2018. All patients presented without a history of pituitary surgery or radiotherapy. All participants provided written informed consent prior to the study.

\section{Acquisition Protocol}

Participants were scanned using a 7T whole-body scanner (Magnetom, Siemens Healthineers). A SC72CD gradient coil was used $\left(\mathrm{G}_{\max }=70 \mathrm{mT} / \mathrm{m}\right.$, maximum slew rate $=200$ tesla $/ \mathrm{m} / \mathrm{sec}$ ), with a single-channel transmit and 32-channel receive head coil (Nova Medical). Scanning included a T1-weighted MP2RAGE sequence with the following parameters: TE $3.62 \mathrm{msec}$, TR $6000 \mathrm{msec}$,
TI $1050 \mathrm{msec}$ (2nd pulse $3000 \mathrm{msec}$ ), flip angle (FA) $5^{\circ}$ (2nd pulse $4^{\circ}$ ), field of view (FOV) $224 \times 168 \mathrm{~mm}^{2}$, number of slices $=240$, voxel size $0.7 \mathrm{~mm}^{3}$ isotropic, and scan time 8:08 minutes. Coronal oblique T2-weighted turbo spin echo (TE $59 \mathrm{msec}$, TR $6000 \mathrm{msec}$, FA 180 ${ }^{\circ}$, FOV $200 \times 168 \mathrm{~mm}^{2}$, in-plane voxel size $0.4 \times 0.4 \mathrm{~mm}^{2}$, slice thickness $2 \mathrm{~mm}$, number of slices $=60$, time $6: 50$ minutes) and high-angular-resolved DWI $\left(b 1200 \mathrm{~mm}^{2} / \mathrm{sec}\right.$, TE 67.6 msec, TR $7200 \mathrm{msec}$, voxel size $1.05 \mathrm{~mm}^{3}$ isotropic, FA $180^{\circ}$, number of slices $=66$, in-plane acceleration $\mathrm{R}=3$, reversed-phase encoding in anteroposterior and posteroanterior directions for paired acquisition in 64 directions, total acquisition time 18:38 minutes) sequences were prescribed. Dielectric pads and localized shimming methods were employed to reduce signal artifact at the skull base.

\section{Image Analysis}

Motion-probing gradient DWI as well as B0 images were postprocessed with MRtrix3 (Brain Research Institute) to derive tensor images for each participant. ADC maps were generated from the tensor images using the default tensor2metric function in MRtrix3. Regions of interest (ROIs) were drawn over the tumor on each slice for which the tumor was visible on the ADC image in the coronal plane. ROIs were drawn conservatively to avoid tumor boundaries and minimize partial volume effects. Areas with increased signal distortion were excluded from ROI measures to reduce the role of air-tissue interface artifacts in the analysis. The average ADC of the entire tumor was calculated using MRtrix3. Equivalent ovoid ROIs were placed in the gray matter of the left and right temporal lobes on the slice with the greatest tumor cross-sectional area to accommodate for any $\mathrm{B} 0$ field inhomogeneity. The mean tumor ADC was divided by the averaged $\mathrm{ADC}$ value of the left and right temporal gray matter to yield a ratio of tumor ADC to gray matter ADC (Fig. 1).

\section{Intraoperative Ratings and Histopathological Analysis}

All patients underwent ETS performed by a single neurosurgeon, and an intraoperative tumor consistency rating system similar to a previously reported method was employed. Tumor consistency was rated based on intraoperative observation by the surgeon on a scale from 1 (least firm) to 5 (most firm) using objective criteria established by Zada et al. for grading meningioma consistency. ${ }^{35}$ The grading criteria were as follows: grade 1, extremely soft tumor; grade 2, soft tumor, bulk of tumor freely suctioned, and fibrous stroma remains; grade 3, average consistency and cannot be freely suctioned, and requires some degree of mechanical debulking; grade 4, firm tumor, requires mechanical debulking; and grade 5, extremely firm and/ or calcified tumor, often requiring en bloc resection..$^{35}$ The surgeon was blinded to the 7T DWI results in this study at the time during which consistency was rated. The extent of resection was determined by intraoperative gross inspection and confirmed by postoperative MRI.

Histopathological analyses were performed by a neuropathologist who was blinded to the MRI findings and intraoperative tumor consistency ratings. Cellularity was 

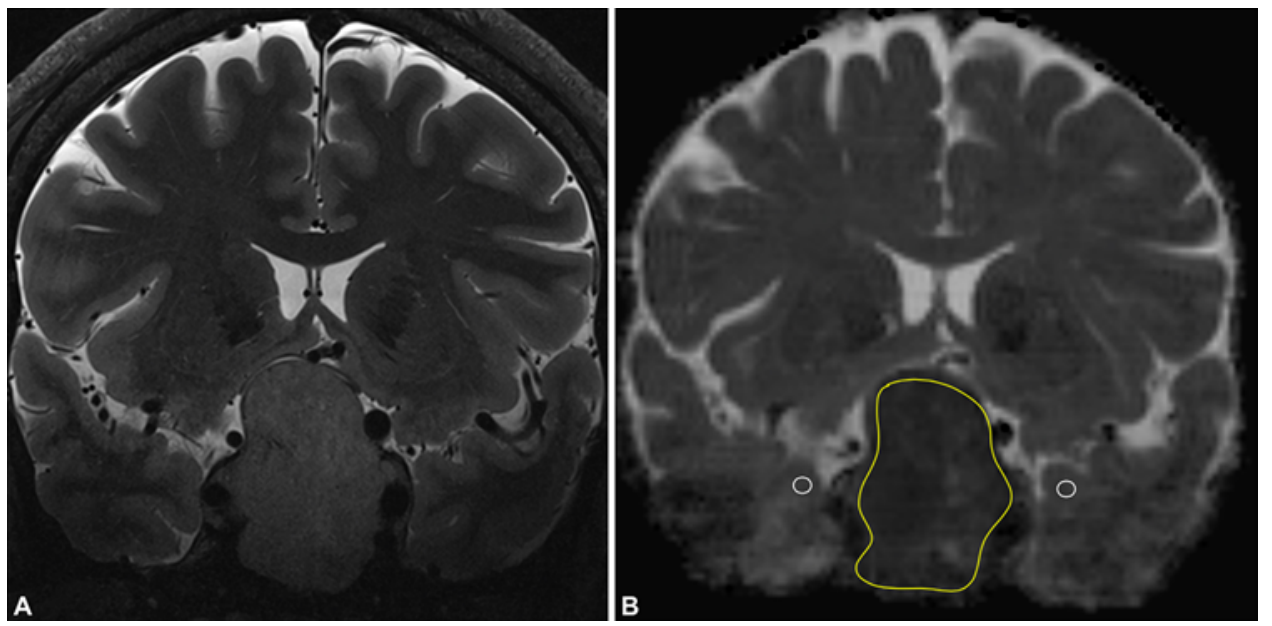

FIG. 1. A: A T2-weighted turbo spin echo image of a patient with pituitary macroadenoma. B: An ADC image with the pituitary tumor ROI in yellow and left and right temporal gray matter ROIs marked in white. Figure is available in color online only.

quantified on a $\times 100$ field using $\mathrm{H} \& \mathrm{E}$ with the following parameters: 1, 1-1000 cells; 2, 1000-5000 cells; or 3, > 5000 cells. Vascularity was quantified using CD31 staining in a $\times 100$ field with the following parameters: 0 , no vascularity; $1,<10$ vessels; $2,10-20$ vessels; or 3, > 20 vessels. Collagen content was evaluated using trichrome stain on a $\times 100$ field using the following parameters: 0 , $0 \%$ blue; $1,<50 \%$ pale blue or $<4 \%$ dark blue; $2,>50 \%$ pale blue or $<50 \%$ dark blue; or $3,>50 \%$ dark blue (Fig. 2). Pathology specimens that were primarily composed of fibroconnective tissue or were obscured by blood products were excluded from histopathological analysis.

\section{Statistical Analysis}

Analyses among each of the imaging or histological features were performed using the following: univariate analysis using Pearson correlations, chi-square test, or independent-samples t-tests, where appropriate. For dependent variables nonnormally distributed, analysis was performed using nonparametric Mann-Whitney U-tests. Receiver operating characteristic (ROC) curve analyses were performed to assess for optimal ADC threshold to predict tumor consistency and extent of resection. For the purposes of the ROC analysis, an intraoperative firmness rating of $0-2$ was considered "soft" and 3-5 was considered "firm." A p value $<0.05$ was considered to be statistically significant. All analyses were performed utilizing a standard statistical package (SPSS V22.0, IBM Corp.). Mean values are presented \pm SD.

\section{Results}

Thirteen patients with pituitary macroadenoma underwent DWI as part of an optimized skull base imaging 7T MRI protocol. All patients underwent ETS, during which tumor firmness was assessed by a single neurosurgeon. All surgical samples were evaluated by an experienced neuropathologist. Patient demographic and disease features are shown in Table 1. Imaging, surgical, and histopathological tumor properties are shown in Tables 2 and 3.

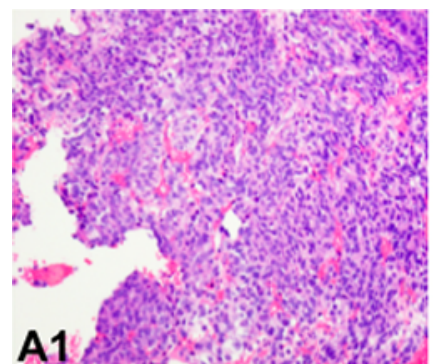

3

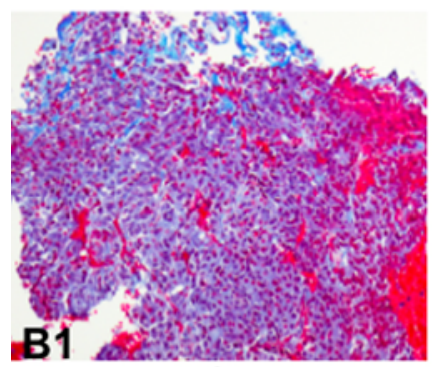

1

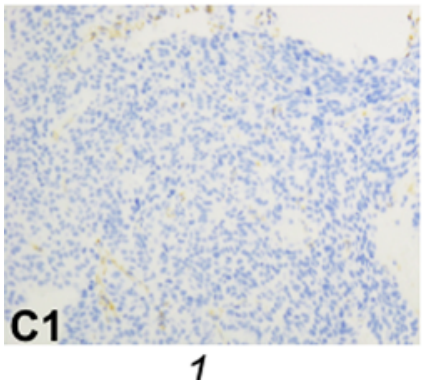

1

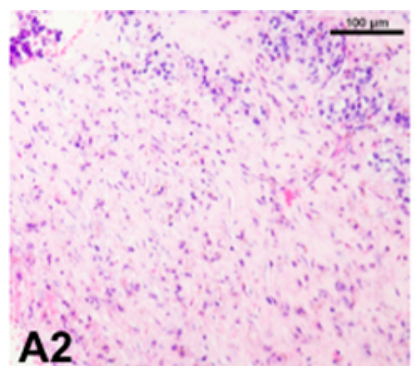

2

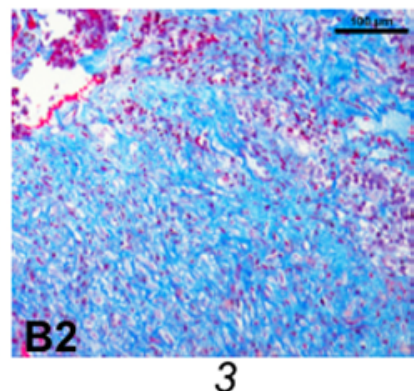

3

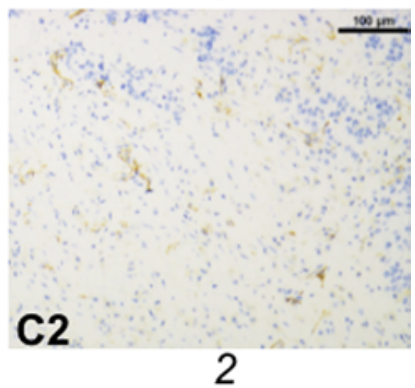

FIG. 2. Histopathology scoring criteria. A1 and A2: Cellularity was assessed on $\mathrm{H}$ \& E-stained photomicrographs. B1 and B2: Collagen content was assessed with trichrome-stained photomicrographs. $\mathrm{C} 1$ and C2: Vascularity was assessed with CD31-stained photomicrographs. Bar $=100 \mu \mathrm{m}$. Figure is available in color online only. 
TABLE 1. Demographic and disease features of 13 patients with pituitary adenoma

\begin{tabular}{lc}
\hline \multicolumn{1}{c}{ Feature } & Value \\
\hline Demographics & $7(53.8 \%)$ \\
\hline Male & $42.3(14.3)$ \\
\hline Age, yrs & $14.4(4.2)$ \\
\hline Disease features & $3.2(2.2)$ \\
\hline Tumor volume, $\mathrm{cm}^{3}$ & $3(23.1 \%)$ \\
\hline Tumor transverse diameter, cm & $48.7(11.2)$ \\
\hline Secreting
\end{tabular}

Values are presented as the mean (SD) or as the number (\%) of patients.

\section{Tumor ADC and Surgical Outcomes}

The mean raw tumor ADC value was $7.67 \times 10^{-4} \mathrm{~mm}^{2} /$ sec $\pm 3.99 \times 10^{-4} \mathrm{~mm}^{2} / \mathrm{sec}$, with a range from $2.81 \times 10^{-4}$ $\mathrm{mm}^{2} / \mathrm{sec}$ to $1.6 \times 10^{-3} \mathrm{~mm}^{2} / \mathrm{sec}$. The mean corrected tumor ADC ratio was $1.22 \pm 0.57$ (range $0.46-2.36$ ). The mean intraoperative tumor consistency rating was $2.31 \pm$ 1.11. Corrected ADC and intraoperative consistency were negatively correlated $(r=-0.6, p=0.029)$. Gross-total resection was achieved in $7(53.8 \%)$ of 13 patients. The mean corrected ADC value of tumors that were gross totally resected $(1.54 \pm 0.52)$ was significantly greater than that of those that were subtotally resected $(0.85 \pm 0.37)(p=0.02)$. These results are shown in Fig. 3.

\section{Tumor ADC and Histopathology}

One trichrome stain and two CD31 stains were excluded from analysis due to poor specimen quality. The mean tumor cellularity rating was $2.33 \pm 0.49$. No tumors received a cellularity rating of less than 2 . The mean ADC value of tumors with a cellularity rating of 2 (moderate) was greater than that of tumors with a cellularity rating of 3 (high) $(1.51 \pm 0.51$ vs $0.8 \pm 0.38$, respectively; $p=$ $0.035)$. The mean tumor collagen content rating was 1.92 \pm 0.9 (range $1-3$ ). The mean tumor ADC was negatively correlated with collagen content $(r=-0.72, p=0.009)$. The mean tumor vascularity rating was $2.36 \pm 0.67$ (range

TABLE 2. Imaging, surgical, and pathological features of 13 pituitary adenomas

\begin{tabular}{cl}
\hline \multicolumn{1}{c}{ Feature } & Value \\
\hline Imaging & \\
\hline Corrected tumor ADC & $1.22(0.57)$ \\
\hline Surgical & $7(53.8 \%)$ \\
\hline Gross-total resection & $2.31(1.11)$ \\
\hline Firmness rating & \\
\hline Pathology rating & $2.33(0.49)$ \\
\hline Cellularity & $1.92(0.9)$ \\
\hline Collagen content & $2.36(0.67)$ \\
\hline Vascularity
\end{tabular}

Values are presented as the mean (SD) or as the number (\%) of patients.
1-3). The mean tumor ADC was not significantly associated with tumor vascularity $(r=-0.09, \mathrm{p}>0.05)$. These results are shown in Fig. 4.

\section{Tumor Histopathology and Surgical Outcomes}

The intraoperative firmness rating was positively correlated with both the degree of tumor staining on trichrome $(\mathrm{r}=0.60, \mathrm{p}=0.04)$ and cellularity $(\mathrm{r}=0.586, \mathrm{p}=0.045)$. No relationship was observed between the firmness rating and vascularity $(\mathrm{r}=0.19, \mathrm{p}>0.05)$. There was a trend toward tumors subtotally resected to exhibit a greater intraoperative tumor firmness rating ( 3 vs $1.7 ; \mathrm{p}=0.051$ ). The degree of trichrome staining was correlated positively with tumor firmness $(r=0.60, p=0.04)$.

Cellularity did not differ significantly between tumors that were gross totally resected $(2.16 \pm 0.41)$ and those that were subtotally resected $(2.5 \pm 0.54)(\mathrm{p}>0.05$. Tumors that were gross totally resected had a significantly lower collagen content $(1.17 \pm 0.41)$ than those that were subtotally resected $(2.67 \pm 0.51)(\mathrm{p}=0.004)$. Vascularity did not differ between gross totally resected tumors $(2.4 \pm 0.55)$ and subtotally resected tumors $(2.33 \pm 0.82)(\mathrm{p}>0.05)$.

\section{ROC Curve Analysis}

We found that the optimal threshold of an ADC ratio value above 0.87 corresponded with a sensitivity of $80 \%$ and a specificity of $100 \%$ (area under the curve [AUC] $0.90, \mathrm{p}=0.043$ ). A value above this threshold corresponded with soft tumors and below this threshold corresponded with firm tumors. The optimal cutoff for distinguishing gross-total versus subtotal resection was an ADC value of 1.19 , such that ADC values below this cutoff were predictive of subtotal resection with an optimal sensitivity of $85.7 \%$ and specificity of $83.3 \%$ (AUC $0.833, \mathrm{p}=0.046$ ). These results are shown in Fig. 5.

\section{Discussion}

Preoperative indicators of tumor consistency are valuable for surgical planning and predicting the extent of resectability of pituitary adenomas. Few studies have investigated ADC as an imaging method for predicting tumor firmness; however, conflicting results have limited the application of this modality in clinical practice. ${ }^{9,19,23,33}$ Here, we employ 7T DWI to acquire high-spatial resolution ADC maps and correlate imaging findings with intraoperative tumor firmness ratings and histopathology findings for pituitary adenomas.

\section{Tumor Features That Limit the Extent of Pituitary Adenoma Resection}

Pituitary adenomas typically exhibit an indolent disease course and are usually well controlled by surgical intervention. ETS has become the preferred technique for pituitary surgery as a result of increased field visualization and reduced need for invasive procedures. However, a number of inherent tumor-related features can preclude gross-total resection and potentially compromise surgical outcomes from diverse causes such as sustained compression of the optic apparatus, postoperative hemorrhage, and 
TABLE 3. Tumor ADC ratio, intraoperative firmness rating, extent of resection, and functional status of the patients' tumors

\begin{tabular}{ccccc}
\hline Subject No. & Tumor ADC Ratio & Tumor Consistency Rating* & Extent of Resection & Functional Status \\
\hline 1 & 0.4645 & 5 & Subtotal & Nonsecreting \\
\hline 2 & 1.0433 & 2 & Subtotal & Nonsecreting \\
\hline 3 & 0.6987 & 2 & Subtotal & Nonsecreting \\
\hline 4 & 0.7031 & 4 & Subtotal & Prolactin \\
\hline 5 & 0.6391 & 2 & Gross & Nonsecreting \\
\hline 6 & 0.6679 & 3 & Subtotal & GH \\
\hline 7 & 2.3627 & 2 & Gross & Nonsecreting \\
\hline 8 & 1.5072 & 2 & Subtotal & Nonsecreting \\
\hline 9 & 1.8018 & 1 & Gross & FSH \\
\hline 10 & 1.3611 & 1 & Gross & Nonsecreting \\
\hline 11 & 1.3400 & 2 & Gross & Nonsecreting \\
\hline 12 & 1.6822 & 2 & Gross & Nonsecreting \\
\hline 13 & 1.6267 & 2 & Gross & Nonsecreting \\
\hline
\end{tabular}

FSH = follicle-stimulating hormone; $\mathrm{GH}=$ growth hormone.

* The consistency was rated on a scale from 1 to 5 , with 1 indicating the tumor was least firm and 5 indicating the tumor was most firm.

the need for a second procedure. ${ }^{18}$ Tumor qualities such as large volume, ${ }^{16}$ significant suprasellar or lateral extension,,$^{1,5}$ dumbbell/hourglass shape, ${ }^{5,22}$ and firmness ${ }^{5,10}$ can lead to multiple staged endoscopic surgeries or to the need for transcranial approaches. Tumor volume has been shown to be a significant predictor of gross-total resection, with reported rates of gross-total resection of giant pituitary adenoma ranging from $20.4 \%{ }^{16}$ to $47.2 \%{ }^{15}$ following ETS, compared with overall pituitary adenoma resection rates of approximately $74 \% .{ }^{6}$ Irregular tumor shape can also limit the extent of resection. Pituitary adenomas that are bi-lobed and constricted at the level of the sellar diaphragm are descried as having an "hourglass" or "dumbbell" shape. ${ }^{5,18}$ These tumors are particularly difficult to resect endoscopically, especially in the presence of an intrasellar component. ${ }^{5,22}$ Tumors that extend to sensitive neurovascular structures, including the internal carotid arteries, ${ }^{24}$ cranial nerves, ${ }^{2}$ and cavernous sinuses, ${ }^{21,31}$ also commonly preclude complete resection.

\section{Radiological Markers of Pituitary Adenoma Firmness}

In addition, tumor consistency is an important factor that can affect the extent of resection and inform the choice in surgical approach. With loss of the reticulin and acinar structure of the normal pituitary gland, many soft tumors can be effectively removed using aspiration and curettage. ${ }^{32}$ However, in the 5\%-13\% of pituitary adenomas in which excess collagen deposition contributes to a firm or fibrous texture, resection can be technically challenging when performing ETS. ${ }^{14,27,32}$ Recently, various
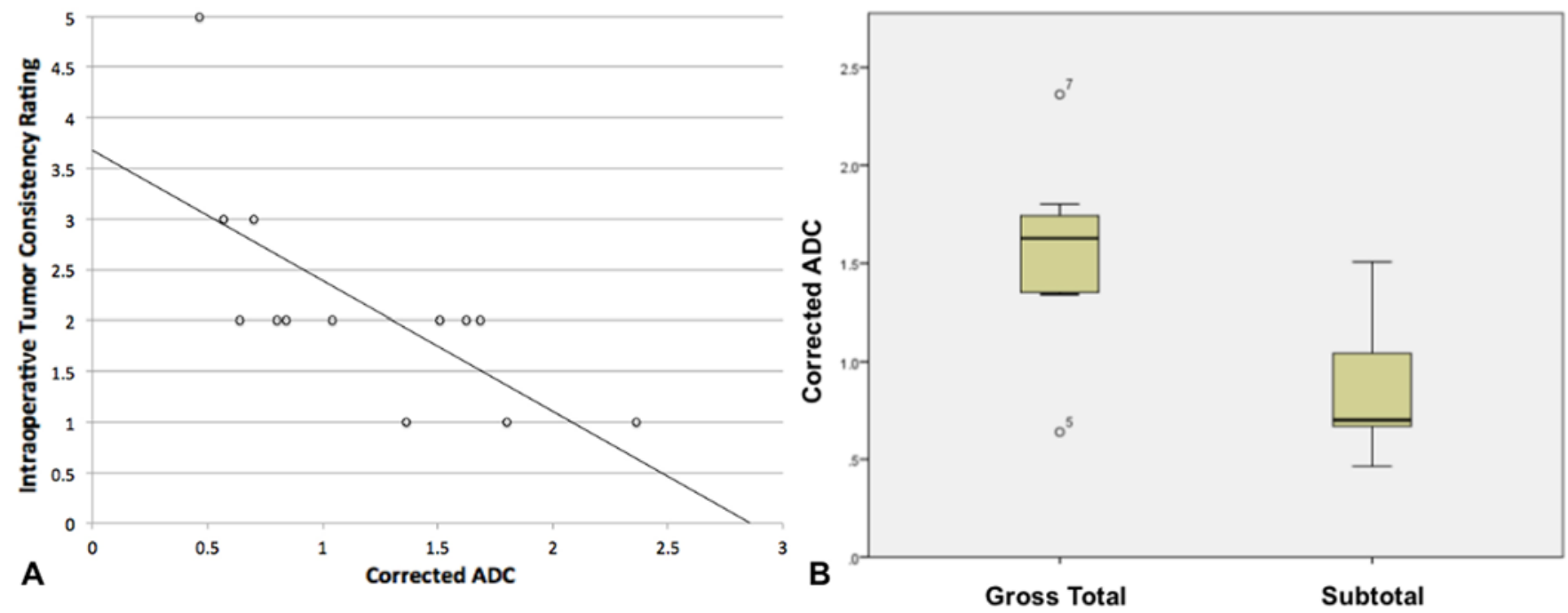

FIG. 3. A: Pearson correlation showing a significant negative correlation between corrected tumor ADC values and intraoperative tumor consistency rating. Circles represent tumors. B: Plot showing corrected tumor ADC values of pituitary adenomas that were subtotally and gross totally resected. Bars indicate the maximum and minimum values. The two circles represent outliers. Figure is available in color online only. 

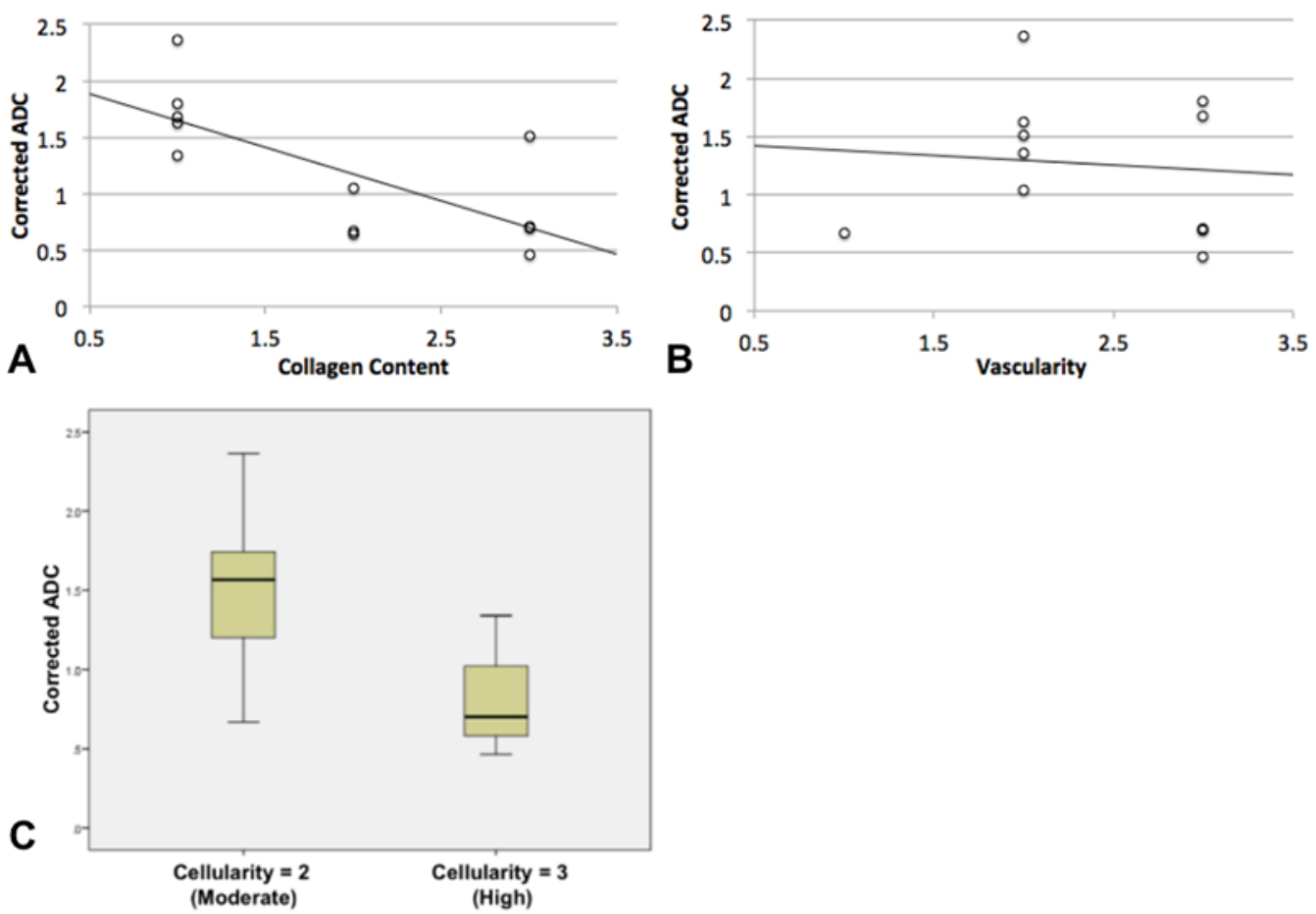

FIG. 4. A: Pearson correlation showing a significant negative correlation between corrected tumor ADC values and tumor collagen content. B: Pearson correlation showing a nonsignificant negative correlation between corrected tumor ADC values and tumor vascularity. C: Plot showing higher corrected tumor ADC in tumors with moderate (a rating of 2) compared with high (rating of 3 ) cellularity. Figure is available in color online only.

MRI modalities have been explored as noninvasive methods of assessing the consistency of pituitary adenomas in order to guide surgery and predict prognosis. A lower T2weighted tumor signal has been found to correlate with higher collagen content and more fibrous consistency. ${ }^{14,27}$ Yamamoto et al. used 3T contrast-enhanced FIESTA to show that firm tumors had a lower tumor-to-healthy white matter T2 signal intensity ratio than soft tumors, concluding that advanced imaging modalities may provide more precise information about pituitary adenoma tissue properties than that provided by conventional MRI sequences. ${ }^{32}$ However, other studies have failed to find imaging markers that significantly correlate with the consistency of pituitary adenomas. To this point, the ability to accu-
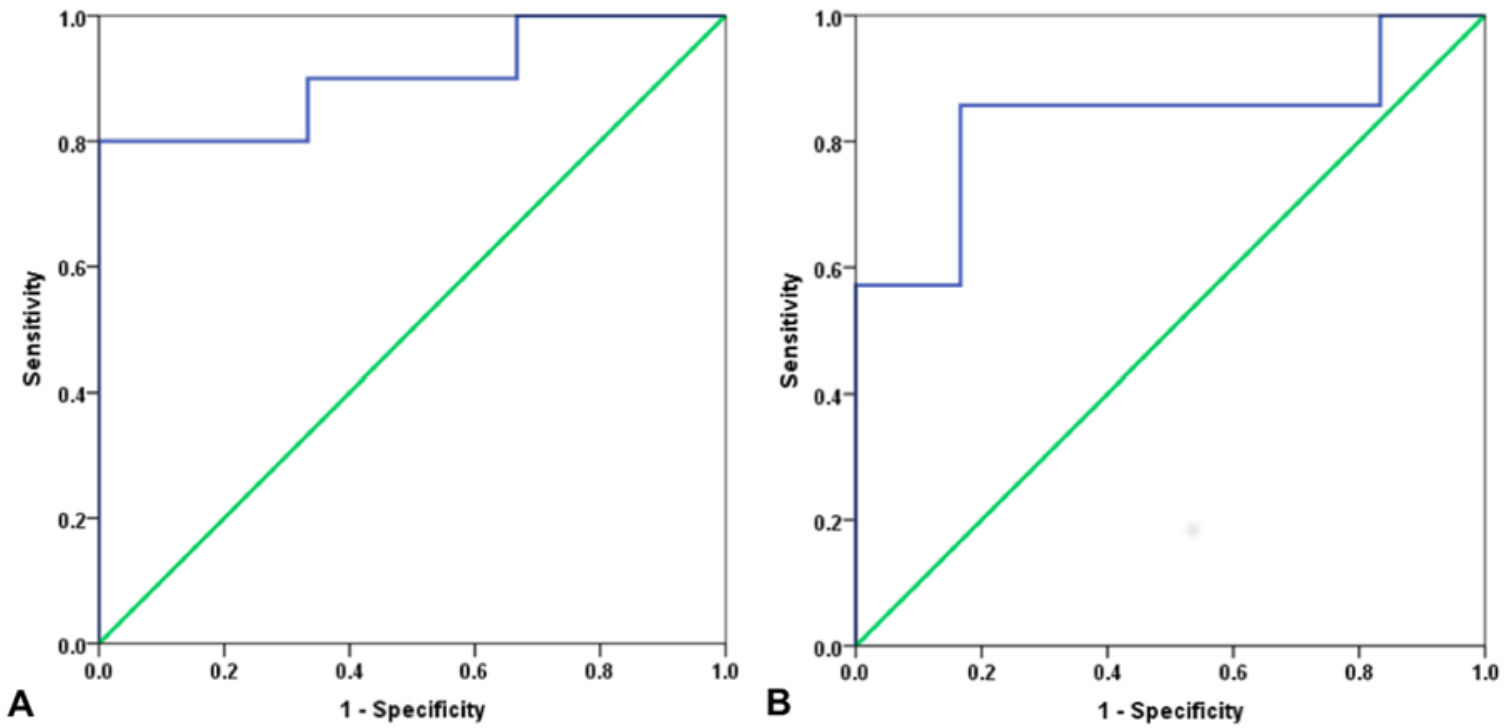

FIG. 5. A: ROC curve analysis of tumor ADC ratio and intraoperative tumor firmness rating: sensitivity $80 \%$, specificity $100 \%$, AUC $0.90 ; p=0.044$. B: ROC curve analysis of tumor ADC ratio and the extent of resection: sensitivity $85.7 \%$, specificity $83.3 \%$, AUC $0.833 ; p=0.046$. Figure is available in color online only. 
rately predict tumor firmness using radiological markers remains controversial. ${ }^{7,13}$

\section{Utility of Diffusion-Weighted Imaging in Characterizing Pituitary Adenoma Consistency}

DWI is an MRI modality that may confer several advantages over conventional imaging in the characterization of tumor consistency. DWI is sensitive to molecular water motion, making it a valuable tool for studying tissue cellularity, neuronal membrane integrity, and other biological properties that influence water movement. ${ }^{8} \mathrm{ADC}$ is a DWI-derived metric that quantifies the overall water molecule diffusion within a voxel, with lower ADC values indicating restricted water diffusion. ${ }^{8}$ Because restrictions in intra- and extracellular water diffusion may also contribute to global tumor properties, ADC is a potentially valuable measure of tumor microstructure and consistency. However, the ability of tumor ADC to predict pituitary adenoma consistency is controversial, and few studies have examined this application of DWI. Pierallini et al. reported that softer pituitary adenomas tended to have more restricted water diffusion and lower resultant ADC values. ${ }^{23}$ More recently, Boxerman et al. found the opposite result, with softer tumors exhibiting higher ADC values and, furthermore, that a high ADC value was associated with gross-total resection. ${ }^{9}$ Additionally, the ADC value was shown to be negatively correlated with the presence of reticulin fibers histologically. ${ }^{9}$ Suzuki et al. used linescan DWI to show that higher ADC values are associated with soft adenomas compared with tumors of intermediate firmness, but this effect was not significant. ${ }^{29}$ Most recently, Alimohamadi et al. showed that secreting pituitary adenomas with low ADC values are more likely to be fibrous and unamenable to suctioning. ${ }^{4}$

Given the considerable inconsistency in the literature, the present study aimed to elucidate the association between ADC and pituitary adenoma consistency using an advanced 7T MRI protocol, optimized for imaging the skull base. While DWI is a powerful tool for measuring quantitative properties of biological tissue, the low spatial resolution of DWI sequences acquired at conventional field strengths can lead to a loss of subtle architectural detail from voxel averaging. ${ }^{3}$ Importantly, the previously discussed studies each used $1.5 \mathrm{~T}$ systems and all had relatively large DWI voxel sizes of approximately $8 \mathrm{~mm}^{3}$, and volume averaging may have biased prior correlations between $\mathrm{ADC}$ and tumor consistncy. ${ }^{9,23,29}$ DWI acquisition in the study by Pierallini et al. employed a 5-mm slice thickness with a 1-mm interslice gap. ${ }^{23}$ The slice thickness in the study by Suzuki et al. was $3 \mathrm{~mm}$ with a $0.5-\mathrm{mm}$ interslice gap. ${ }^{29}$ The DWI voxel sizes in the study by Boxerman et al. were the smallest of the three studies with a 3-mm slice thickness and no interslice gap. ${ }^{9}$ The DWI spatial resolution in the present study $(1.05 \times 1.05 \times 1.05 \mathrm{~mm})$ yielded voxel sizes that were nearly eight-fold smaller than those seen in previous studies, and we believe that the higher SNR and smaller voxel size attainable with 7T MRI may clarify the value of ADC in predicting the consistency of pituitary adenomas preoperatively. While there have not been any studies to examine the use of ADC values in predicting pituitary adenoma firmness at 3T, DWI resolution remains limited at clinical field strengths, and 7T DWI permits smaller voxel sizes and a greater SNR than can be attained using 3T imaging, making ultra-high field DWI a well-suited modality for clarifying the role of preoperative ADC measurement in predicting tumor consistency.

Tumor collagen content and cellularity are important determinants of tumor consistency. High cellularity and high nucleus-to-cytoplasm ratio are factors that are associated with firm pituitary adenomas. This principle is supported by reports of lower T2 signal intensity, and presumably reduced fluid content, in firm pituitary adenomas. ${ }^{14,27}$ Properties that determine tumor consistency can also affect water diffusion within biological tissue. Increased cellularity is associated with decreased ADC because water diffusion in the interstitium is less restricted than water diffusion intracellularly. ${ }^{17,28}$ The lower ADC values observed in firmer tumors in this study may also reflect higher cellularity and higher nucleus-to-cytoplasm ratio, as these features represent physical barriers to molecular water movement and restrict ADC within a voxel. This is supported by our finding that tumors with higher cellularity exhibited lower ADC ratios in the present study.

$\mathrm{ADC}$ has also been explored as a metric to predict tumor consistency in other intracranial tumors, including meningiomas and gliomas. Yogi et al. found that firm meningiomas exhibited significantly lower ADC values compared with soft meningiomas. ${ }^{34}$ Similarly, Sugahara et al. found that glioma cellularity negatively correlated with tumor ADC. ${ }^{28} \mathrm{ADC}$ of hepatic lesions has also been shown to negatively correlate with degree of fibrosis. ${ }^{12}$ Our results also suggest that ADC is inversely related to biological factors that contribute to tumor firmness, namely, cellularity and fibrous content, which supports prior accounts in various intracranial tumor types including pituitary adenoma. ${ }^{9,28,34}$

\section{Limitations and Future Directions}

The present study has several limitations. Firstly, DWI is susceptible to main magnetic field (B0) inhomogeneity and off-resonance effects near air-tissue interfaces, such as the paranasal sinuses. ${ }^{11}$ Patients with microadenomas were excluded from this study in part as a result of DWI spatial resolution limitations, which remain even at 7T, and also as a result of high susceptibility artifact in this region that can reduce ADC image quality in these small tumors. Several maneuvers mitigated these artifacts. Previous work has suggested that ROI placement of the pituitary tumor in the coronal plane may reduce ADC artifact from the paranasal sinuses. ${ }^{9}$ Unlike previous studies, ours employed high-resolution isotropic DWI image acquisition that allowed tumor ROI placement in the coronal plane. To normalize ADC values, we generated ADC ratios (tumor ADC/temporal gray matter ADC). Previous studies have also reported pituitary adenoma signal properties in reference to adjacent healthy tissue, and we believe that this standardization helped mitigate the effects of field inhomogeneity in this region. ${ }^{9,29,32}$ Lastly, localized shimming techniques and dialectic pads were used to minimize image distortion in this study. However, the effects of B0 inhomogeneity are inherent limitations of performing DWI at the skull base, and technical development will help to 
minimize these artifacts even further and permit this technique to also be applied to pituitary microadenomas.

The histopathological analysis in this study is another potential source of error. Specimens that were obscured by blood products or were primarily composed of connective tissue or normal gland were excluded from the analysis. Pituitary adenomas are resected in a piecemeal manner by suction/aspiration during ETS, and determining the origin of histological samples on imaging, while preserving surgical workflow, is challenging. This reflects an inherent limitation of studies that correlate radiological findings with histological specimens procured endoscopically. Therefore, histological data from one region of the tumor were correlated with the average ADC of the tumor overall. Because the site of histological evaluation may not be representative of the entire tumor, this methodology may impair our ability to detect correlations between imaging and histopathology. Until ETS methods more reliably localize endoscopic specimens, outcome-based metrics such as the extent of resection are useful in studying the roles of ADC and other MRI measures in predicting tumor consistency.

Our relatively small sample size may have also limited the detectability of findings. The sample size was limited by more stringent inclusion criteria for scanning patients at ultra-high field MRI, exclusion of patients with inadequate DWI image quality, and exclusion of patients who did not progress to surgery or did not have appropriate histological specimens. However, because of the increased sensitivity of 7T DWI in quantifying subtle microstructural alterations in biological tissue, we believe that these preliminary data represent accurate associations between the ADC and intrinsic tumor features. These results may be useful in establishing a consensus with regard to the role of high-resolution preoperative DWI in predicting pituitary adenoma firmness, especially with regard to the conflicting findings seen in previous lower field studies. $9,23,29$

Lastly, we used a relatively subjective single-surgeon intraoperative consistency rating system, which is similar to those used in prior investigations..$^{26,35}$ We used criteria proposed by Zada et al. for grading meningioma consistency in order to standardize these measurements. ${ }^{35}$ While we believe that having a single surgeon grade tumor firmness provided some degree of uniformity to the tumor rating, the subjective nature of this evaluation warrants repeated investigation with firmness ratings from multiple surgeons.

Novel imaging modalities such as MR elastography may also play an important role in predicting pituitary adenoma consistently and should be areas of future investigation. While this technology requires specialized hardware that is not widely used in clinical practice, limiting its widespread applicability at this time, preliminary results have shown that MR elastography may provide advanced information about tissue biology, ${ }^{25}$ and this modality is a promising line of investigation for the noninvasive characterization of tumor firmness.

\section{Conclusions}

The purpose of the present study was to leverage high- spatial resolution 7T DWI and correlate tumor ADC with an intraoperative firmness rating, histopathology, and an outcome-based metric in order to clarify the role of DWI in predicting pituitary macroadenoma consistency. The accurate prediction of tumor consistency is especially important in the context of complex pituitary surgery, as fibrous tumor features may limit endoscopic endonasal resection, and preoperative characterization of firmness may aide in the planning of the surgical route and in predicting operative morbidity and the extent of resection. Previous studies have been limited by low field MRI with large DWI voxel sizes and have yielded conflicting results of ADC as an indicator of pituitary adenoma consistency., ${ }^{9,23,29}$ Our results are in agreement with those reported by Boxerman et al. and suggest that softer tumors exhibit higher average ADC ratios and are more easily completely resected. ${ }^{9}$ High-spatial resolution 7T DWI is a useful noninvasive tool that may allow preoperative assessment of tumor consistency, optimizing risk assessment and surgical planning, and predicting prognosis in patients with pituitary macroadenomas.

\section{Acknowledgments}

Funding was provided by NIH grant R01 CA202911 and the Icahn School of Medicine Capital Campaign, Translational and Molecular Imaging Institute, and Department of Radiology, Icahn School of Medicine at Mount Sinai.

\section{References}

1. Abe T, Iwata T, Kawamura N, Izumiyama H, Ikeda H, Matsumoto K: Staged transsphenoidal surgery for fibrous nonfunctioning pituitary adenomas with suprasellar extension. Neurol Med Chir (Tokyo) 37:830-837, 1997

2. Ahmadi J, North CM, Segall HD, Zee CS, Weiss MH: Cavernous sinus invasion by pituitary adenomas. AJR Am J Roentgenol 146:257-262, 1986

3. Alexander AL, Hasan KM, Lazar M, Tsuruda JS, Parker DL: Analysis of partial volume effects in diffusion-tensor MRI. Magn Reson Med 45:770-780, 2001

4. Alimohamadi M, Sanjari R, Shirani M, Alikhani F, Amirjamshidi A: Initial experience with diffusion-weighted imaging to predict the tumor consistency and surgical success in solid growth hormone producing pituitary macroadenomas. Asian J Neurosurg 14:698-701, 2019

5. Alleyne CH Jr, Barrow DL, Oyesiku NM: Combined transsphenoidal and pterional craniotomy approach to giant pituitary tumors. Surg Neurol 57:380-390, 2002

6. Almutairi RD, Muskens IS, Cote DJ, Dijkman MD, Kavouridis VK, Crocker E, et al: Gross total resection of pituitary adenomas after endoscopic vs. microscopic transsphenoidal surgery: a meta-analysis. Acta Neurochir (Wien) 160:10051021, 2018

7. Bahuleyan B, Raghuram L, Rajshekhar V, Chacko AG: To assess the ability of MRI to predict consistency of pituitary macroadenomas. Br J Neurosurg 20:324-326, 2006

8. Bammer R: Basic principles of diffusion-weighted imaging. Eur J Radiol 45:169-184, 2003

9. Boxerman JL, Rogg JM, Donahue JE, Machan JT, Goldman MA, Doberstein CE: Preoperative MRI evaluation of pituitary macroadenoma: imaging features predictive of successful transsphenoidal surgery. AJR Am J Roentgenol 195:720-728, 2010

10. Cappelletti M, Ruggeri AG, Spizzichino L, D’Amico A, D'avella E, Delfini R: Fibrous pituitary macroadenomas: 
predictive role of preoperative radiologic investigations for proper surgical planning in a cohort of 66 patients. World Neurosurg 121:e449-e457, 2019

11. Deoni SCL: Correction of main and transmit magnetic field (B0 and B1) inhomogeneity effects in multicomponent-driven equilibrium single-pulse observation of T1 and T2. Magn Reson Med 65:1021-1035, 2011

12. Fujimoto K, Tonan T, Azuma S, Kage M, Nakashima O, Johkoh T, et al: Evaluation of the mean and entropy of apparent diffusion coefficient values in chronic hepatitis C: correlation with pathologic fibrosis stage and inflammatory activity grade. Radiology 258:739-748, 2011

13. Hagiwara A, Inoue Y, Wakasa K, Haba T, Tashiro T, Miyamoto $\mathrm{T}$ : Comparison of growth hormone-producing and non-growth hormone-producing pituitary adenomas: imaging characteristics and pathologic correlation. Radiology 228:533-538, 2003

14. Iuchi T, Saeki N, Tanaka M, Sunami K, Yamaura A: MRI prediction of fibrous pituitary adenomas. Acta Neurochir (Wien) 140:779-786, 1998

15. Komotar RJ, Starke RM, Raper DM, Anand VK, Schwartz TH: Endoscopic endonasal compared with microscopic transsphenoidal and open transcranial resection of giant pituitary adenomas. Pituitary 15:150-159, 2012

16. Koutourousiou M, Gardner PA, Fernandez-Miranda JC, Paluzzi A, Wang EW, Snyderman CH: Endoscopic endonasal surgery for giant pituitary adenomas: advantages and limitations. J Neurosurg 118:621-631, 2013

17. Le Bihan D, Breton E, Lallemand D, Grenier P, Cabanis E, Laval-Jeantet M: MR imaging of intravoxel incoherent motions: application to diffusion and perfusion in neurologic disorders. Radiology 161:401-407, 1986

18. Leung GKK, Law HY, Hung KN, Fan YW, Lui WM: Combined simultaneous transcranial and transsphenoidal resection of large-to-giant pituitary adenomas. Acta Neurochir (Wien) 153:1401-1408, 2011

19. Mahmoud OM, Tominaga A, Amatya VJ, Ohtaki M, Sugiyama K, Sakoguchi T, et al: Role of PROPELLER diffusionweighted imaging and apparent diffusion coefficient in the evaluation of pituitary adenomas. Eur J Radiol 80:412-417, 2011

20. McLaughlin N, Eisenberg AA, Cohan P, Chaloner CB, Kelly DF: Value of endoscopy for maximizing tumor removal in endonasal transsphenoidal pituitary adenoma surgery. J Neurosurg 118:613-620, 2013

21. Micko AS, Wöhrer A, Wolfsberger S, Knosp E: Invasion of the cavernous sinus space in pituitary adenomas: endoscopic verification and its correlation with an MRI-based classification. J Neurosurg 122:803-811, 2015

22. Mortini P, Barzaghi R, Losa M, Boari N, Giovanelli M: Surgical treatment of giant pituitary adenomas: strategies and results in a series of 95 consecutive patients. Neurosurgery 60:993-1004, 2007

23. Pierallini A, Caramia F, Falcone C, Tinelli E, Paonessa A, Ciddio AB, et al: Pituitary macroadenomas: preoperative evaluation of consistency with diffusion-weighted MR imaging-initial experience. Radiology 239:223-231, 2006

24. Rey-Dios R, Payner TD, Cohen-Gadol AA: Pituitary macroadenoma causing symptomatic internal carotid artery compression: surgical treatment through transsphenoidal tumor resection. J Clin Neurosci 21:541-546, 2014

25. Sakai N, Takehara Y, Yamashita S, Ohishi N, Kawaji H, Sameshima T, et al: Shear stiffness of 4 common intracranial tumors measured using MR elastography: comparison with intraoperative consistency grading. AJNR Am J Neuroradiol 37:1851-1859, 2016

26. Smith KA, Leever JD, Chamoun RB: Predicting consistency of meningioma by magnetic resonance imaging. J Neurol Surg B Skull Base 76:225-229, 2015
27. Smith KA, Leever JD, Chamoun RB: Prediction of consistency of pituitary adenomas by magnetic resonance imaging. J Neurol Surg B Skull Base 76:340-343, 2015

28. Sugahara T, Korogi Y, Kochi M, Ikushima I, Shigematu Y, Hirai T, et al: Usefulness of diffusion-weighted MRI with echo-planar technique in the evaluation of cellularity in gliomas. J Magn Reson Imaging 9:53-60, 1999

29. Suzuki C, Maeda M, Hori K, Kozuka Y, Sakuma H, Taki W, et al: Apparent diffusion coefficient of pituitary macroadenoma evaluated with line-scan diffusion-weighted imaging. J Neuroradiol 34:228-235, 2007

30. Thotakura AK, Patibandla MR, Panigrahi MK, Mahadevan A: Is it really possible to predict the consistency of a pituitary adenoma preoperatively? Neurochirurgie 63:453-457, 2017

31. Wang S, Lin S, Wei L, Zhao L, Huang Y: Analysis of operative efficacy for giant pituitary adenoma. BMC Surg 14:59, 2014

32. Yamamoto J, Kakeda S, Shimajiri S, Takahashi M, Watanabe K, Kai Y, et al: Tumor consistency of pituitary macroadenomas: predictive analysis on the basis of imaging features with contrast-enhanced 3D FIESTA at 3T. AJNR Am J Neuroradiol 35:297-303, 2014

33. Yiping L, Ji X, Daoying G, Bo Y: Prediction of the consistency of pituitary adenoma: a comparative study on diffusionweighted imaging and pathological results. J Neuroradiol 43:186-194, 2016

34. Yogi A, Koga T, Azama K, Higa D, Ogawa K, Watanabe T, et al: Usefulness of the apparent diffusion coefficient (ADC) for predicting the consistency of intracranial meningiomas. Clin Imaging 38:802-807, 2014

35. Zada G, Yashar P, Robison A, Winer J, Khalessi A, Mack WJ, et al: A proposed grading system for standardizing tumor consistency of intracranial meningiomas. Neurosurg Focus 35(6):E1, 2013

\section{Disclosures}

Dr. Balchandani (the principal investigator in this study) is a named inventor on patents relating to MRI and radiofrequency pulse design. The patents have been licensed to GE Healthcare, Siemens AG, and Philips International. Dr. Balchandani receives royalty payments relating to these patents. She is a named inventor on patents relating to slice-selective adiabatic magnetization T2-preparation (SAMPA) for efficient T2-weighted imaging at ultra-high field strengths, and methods for producing a semi-adiabatic spectral-spatial spectroscopic imaging sequence and devices thereof. Dr. Bederson (a significant contributor in this study and chair of the Department of Neurosurgery) owns equity in Surgical Theater, LLC (manufacturer of the Surgical Navigation Advanced Platform [SNAP] system).

\section{Author Contributions}

Conception and design: Rutland. Acquisition of data: Rutland, Ranti, Tsankova, Bellaire. Analysis and interpretation of data: Rutland, Tsankova, Bederson, Delman, Shrivastava, Balchandani. Drafting the article: Rutland. Critically revising the article: Rutland, Bederson, Delman, Shrivastava, Balchandani. Reviewed submitted version of manuscript: all authors. Approved the final version of the manuscript on behalf of all authors: Rutland. Statistical analysis: Loewenstern. Study supervision: Delman, Shrivastava, Balchandani.

\section{Correspondence}

John W. Rutland: Translational and Molecular Imaging Institute, Icahn School of Medicine at Mount Sinai, New York, NY. jack. rutland@icahn.mssm.edu. 